\title{
Random walk with random resetting to the maximum
}

\author{
Satya N. Majumdar, ${ }^{1}$ Sanjib Sabhapandit, ${ }^{2}$ and Grégory Schehr ${ }^{1}$ \\ ${ }^{1}$ LPTMS, CNRS, Univ Paris Sud, Université Paris-Saclay, 91405 Orsay, France \\ ${ }^{2}$ Raman Research Institute, Bangalore 560080, India
}

(Dated: June 3, 2022)

\begin{abstract}
We study analytically a simple random walk model on a one-dimensional lattice, where at each time step the walker resets to the maximum of the already visited positions (to the rightmost visited site) with a probability $r$, and with probability $(1-r)$, it undergoes symmetric random walk, i.e., it hops to one of its neighboring sites, with equal probability $(1-r) / 2$. For $r=0$, it reduces to a standard random walk whose typical distance grows as $\sqrt{n}$ for large $n$. In presence of a nonzero resetting rate $0<r \leq 1$, we find that both the average maximum and the average position grow ballistically for large $n$, with a common speed $v(r)$. Moreover, the fluctuations around their respective averages grow diffusively, again with the same diffusion coefficient $D(r)$. We compute $v(r)$ and $D(r)$ explicitly. We also show that the probability distribution of the difference between the maximum and the location of the walker, becomes stationary as $n \rightarrow \infty$. However, the approach to this stationary distribution is accompanied by a dynamical phase transition, characterized by a weakly singular large deviation function. We also show that $r=0$ is a special 'critical' point, for which the growth laws are different from the $r \rightarrow 0$ case and we calculate the exact crossover functions that interpolate between the critical $(r=0)$ and the off-critical $(r \rightarrow 0)$ behavior for finite but large $n$.
\end{abstract}

PACS numbers: 05.40.-a, 02.50.-r,87.23.Ge

\section{INTRODUCTION}

Search problems appear in diverse contexts [1-4] and there have been a recent surge of interests in the physics community in these problems [5]. Search strategies may be either systematic or random. In the systematic strategies, the searcher uses deterministic rules (e.g., 'lawnmower') to find a target. On the other hand, the random search mechanism typically involves two kinds of moves: local steps when the searcher looks for a target, and long-range moves during which the searcher does not look for the target but relocates itself to a different territory. The slow search phase is typically modelled by a diffusion or a random walk. The long-range moves may be modelled depending on the specific application [4].

A particularly simple long-range strategy consists of 'resetting' the searcher to a fixed location (say to the initial starting point) with a finite probability/rate. The rational behind this strategy is that if one does not succeed in finding the target via short-range diffusion, it is better to 'restart' the process, rather than continuing on the short-range moves. The effect of such stochastic resetting was first studied by Manrubia and Zanette [6] in the context of multiplicative processes and a slightly different version was studied later by Gelenbe [7] in the context of network theory. Such 'restart' strategy also plays an important role in randomized search algorithms for combinatorial optimization problems [8, 9].

Recently, a very simple model of a Brownian searcher in presence of stochastic resetting to its initial position with rate $r$ was introduced by Evans and Majumdar [10]. In presence of a nonzero $r$, it was shown that at long times, the probability distribution of the position of the walker reaches a nonequilibrium steady state [10]. The temporal relaxation to this steady state was also studied recently [11] and an interesting dynamical phase transition was found: as time progresses, an inner core region around the resetting point reaches the steady state, while the region outside the core is still transient. The boundaries of the core region grow linearly with time at late times [11].

In presence of resetting with rate $r$, the mean first-passage time to find a target located at the origin, by a searcher starting and resetting to $x_{0}$, was computed exactly [10] and was found to have a minimum at an optimal resetting rate $r^{*}$, thus making the search process efficient in presence of resetting. This conclusion holds in all dimensions [12]. Also, it was proved that this non-equilibrium reset dynamics is more efficient in target search compared to an equilibrium Langevin dynamics in presence of an external potential leading to the same steady state [13].

This simple model of diffusion with stochastic resetting, in the single searcher setting, has been generalized in various ways. For example, when the target as well as the resetting positions are not fixed but drawn from specified probability distributions [14], in presence of partial detection (or absorption) of the target by a searcher [15], when the searcher performs a continuous-time random walk [16] or a Lévy flight instead of a Brownian motion/random walk [17, 18], when the searcher moves in a bounded domain [19] or in the presence of a confining potential [20], when the resetting occurs to any of the previously visited sites with a rate proportional to the number of visits to the site [21] etc. Recently the model of random walks with resetting has also been used to understand the behavior in models of enzymatic reactions in biology [22].

Going beyond the one particle setting, the effect of the resetting mechanism in searching an immobile target has also been studied in presence of multiple, but non-interacting searchers [10]. More recently, the resetting has been studied in spatially extended many-body interacting systems, such as for fluctuating interfaces [11, 23] as well as a class of reactiondiffusion models [24] —in both cases, the natural dynamics of the system is stochastically interrupted by resetting it to the initial configuration at a nonzero rate $r$. A nonzero $r$ leads to new non-equilibrium stationary states in such extended sys- 
tems [11, 23].

In this paper, we consider a model where the searcher remembers the maximum location visited so far and the longrange move consists in resetting to this current maximum. This strategy may be thought as the mixture of the systematic search and the random search. In the systematic search strategy since each location is visited only once, if a target in an already visited place is missed (by an imperfect searcher), it is never going to be detected. In the new strategy discussed in this paper, the searcher revisits already searched locations (with certain probability), but also feels a dynamical bias towards exploring new locations (by resetting to the maximum).

This model may actually be useful in the context of animals searching for food. During the foraging period, it is well known that an animal typically performs a random walk in search of food [25, 26]. It is however quite natural for an intelligent animal (with memory) to remember the already visited (explored) sites and thus to have a natural tendency to relocate once in a while to the frontier between already explored and yet unexplored territories, where the probability of finding food may be higher due to the proximity of the unexplored territory. In a one dimensional setting, the animal, besides short-range diffusion, may relocate with a nonzero probability to the current maximum or to the current minimum, which together constitute the frontier between explored and unexplored territories. In this paper, we consider an even more simplified directed version that has the advantage of being exactly solvable. In our model the animal starting at the origin, besides performing short-range standard random walk, relocates stochastically with a nonzero probability to only the positive side of the frontier, i.e., the farthest visited site so far to the right of the origin (i.e., to the maximum). We will see that despite the fact that the position of the walker evolves via a non-Markovian dynamics (as it remembers the maximum position so far in order to relocate), the model allows for an exact solution and thus provides interesting insights into this long-range search strategy.

The rest of the paper is organized as follows. In Section II we introduce the model precisely and summarize our main exact results. In Section III we derive the generating functions for the probabilities of the position and the maximum after $n$ time steps. We then examine in detail the asymptotic large $n$ statistics in the two opposite limits respectively in the next two sections: (i) the case without resetting but only with diffusion in Section $[\mathrm{IV}$ and (ii) the case where only resetting occurs without any diffusion in Section $\mathrm{V}$ In Section $\mathrm{VI}$ we analyze the situation for arbitrary resetting rate. In Section VII. we analyze the statistics of the maximum and the position in the scaling limit $r \rightarrow 0, n \rightarrow \infty$ while keeping the product $r n$ fixed. The crossover scaling functions are computed exactly in this Section and compared to numerical simulation results. Finally we conclude with a summary and some open questions in Section VIII

\section{THE MODEL AND THE SUMMARY OF MAIN RESULTS}

We consider a walker moving on a one-dimensional lattice, initially starting from the origin. Here each lattice site should be thought of as a 'region' which is much larger than the 'size of the searcher', but much smaller than the whole region. The searcher spends some characteristic time $\tau$ in each region (lattice site), and we consider time steps in units of $\tau$ and take it to be discrete. Let $x(n)$ denote the position of the walker at step $n$ and $m(n)$ denote the current position of the maximum at step $n$, i.e.

$$
m(n)=\max [x(0)=0, x(1), x(2), \ldots, x(n)] .
$$

The position $x(n)$ evolves with time via the following stochastic dynamics. At any given time step $n$, if the position $x(n)$ of the walker is less than the maximum position $m(n)$ reached up to that time (i.e, $x(n)<m(n)$ strictly), then in the next time step, the position is reset to the maximum position with probability $r$. With the remaining probability $(1-r)$, the walker moves either to the right or to the left lattice site, with equal probability $(1-r) / 2$. On the other hand, if $x(n)=m(n)$, then in the next time step, the walker moves either to the right or to the left lattice site with equal probability $1 / 2$. The dynamics is precisely defined by the following evolution rules [see Fig. 1](a)]:

$$
\begin{aligned}
& \text { if } x(n)<m(n) \\
& (x, m) \rightarrow \begin{cases}(x+1, m) & \text { with probability }(1-r) / 2, \\
(x-1, m) & \text { with probability }(1-r) / 2, \\
(m, m) & \text { with probability } r\end{cases}
\end{aligned}
$$

and if $x(n)=m(n)$,

$$
(x, m) \rightarrow \begin{cases}(m+1, m+1) & \text { with probability } 1 / 2, \\ (m-1, m) & \text { with probability } 1 / 2 .\end{cases}
$$

Evidently, the evolution of $x(n)$ is non-Markovian by itself, since the walker has to remember the maximum position reached so far in order to reset. However, the dynamics of the pair of stochastic variables $\{x(n), m(n)\}$ is Markovian in the two dimensional $(x, m)$ plane, and this is the key point behind the solvability of the model. Figure 1 depicts the motion in the $(x, m)$ plane.

It is useful to summarize our main results. Our main objective is to compute the statistics of the two random variables $x(n)$ and $m(n)$. Let us first recall that in the absence of resetting $(r=0)$, the walker performs a standard one dimensional random walk, for which $x(n)$, converges to a Gaussian random variable with zero mean and variance $n$, for large $n$. Hence, the probability distribution of the position converges, for large $n$, to $P_{\mathrm{x}}(x, n) \rightarrow \sqrt{\frac{1}{2 \pi n}} \exp \left[-x^{2} / 2 n\right]$. Similarly, for $r=0$, the distribution of the maximum $m(n) \geq 0$, for large $n$, converges to a half-Gaussian: $P_{\mathrm{m}}(m, n) \rightarrow \sqrt{\frac{2}{\pi n}} \exp \left[-m^{2} / 2 n\right]$ with support only over $m \geq 0$. Thus, for $r=0$, both the position and the maximum typically grow diffusively as $\sqrt{n}$ for large $n$. 


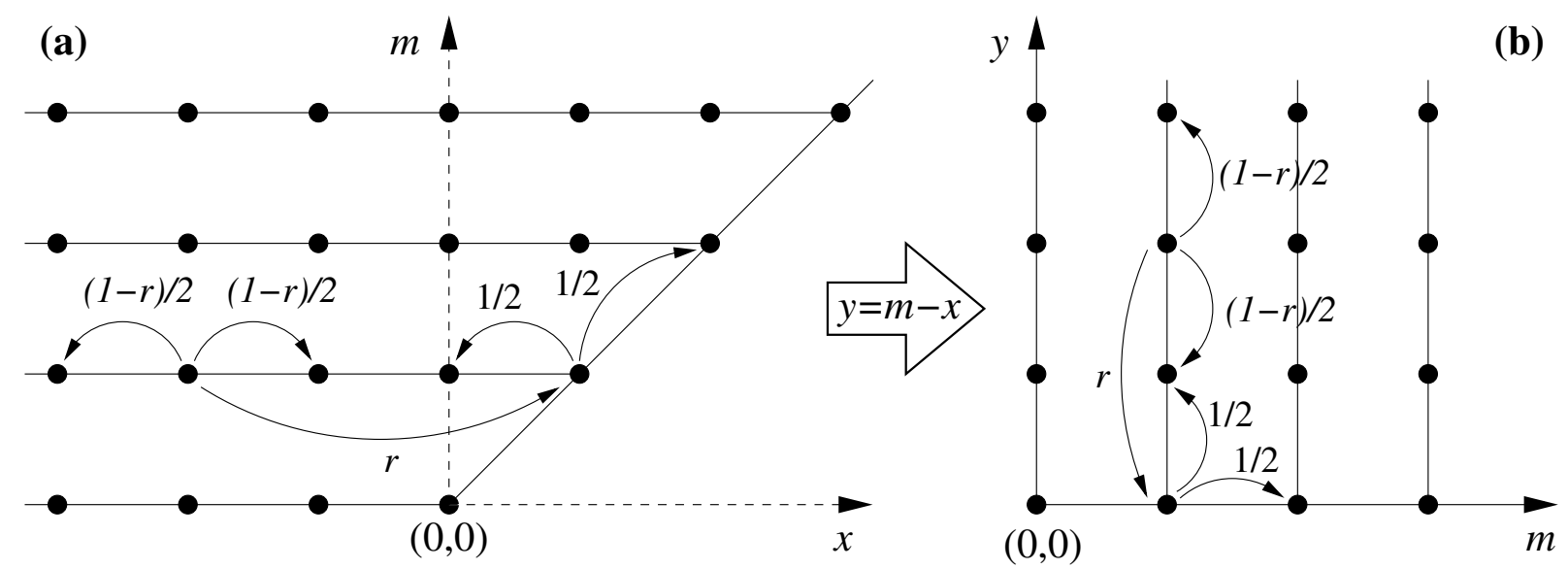

FIG. 1. (a) The allowed lattice points for the walker to move in the $(x, m)$ plane. The walker is restricted to move only along the horizontal (constant $m$ ) lines except when it is on the $x=m$ line. When on the $x=m$ line, the walker can either move to a level up on the same line $(m, m) \rightarrow(m+1, m+1)$ with probability $1 / 2$ or move to the left site while staying on the same level $(m, m) \rightarrow(m-1, m)$ with probability 1/2. (b) The comb lattice structure after the transformation $y=m-x$. In the $(y, m)$ plane, the walker is restricted to move along the vertical lines except when $y=0$. From a point $(0, m)$ the walker can go either to $(0, m+1)$ with probability $1 / 2$ or to $(1, m)$ with probability $1 / 2$.

When the resetting to the maximum is switched on $(r>0)$, the walker feels a dynamical bias towards the maximum. Hence, one expects that both $x(n)$ and $m(n)$ will grow faster than pure diffusion for large $n$. The question is how much faster? We will see that for $r>0$ (strictly), both $m(n)$ and $x(n)$ grow linearly with $n$ for large $n$ with the same speed. In addition, the variance of both $m(n)$ and $x(n)$ grow diffusively for large $n$ with the same diffusion coefficient. This suggests that for all $r>0$, the position latches on to the maximum and indeed, we show that the difference variable $m(n)-x(n)$ approaches a stationary distribution as $n \rightarrow \infty$ for all $r>0$. Our exact results are summarized below.

Statistics of the maximum $m(n)$ : The average maximum, for large $n$, behaves as

$$
\begin{aligned}
\langle m(n)\rangle & \simeq \sqrt{\frac{2 n}{\pi}} & & \text { for } \quad r=0, \\
& \simeq v(r) n & & \text { for } \quad r>0,
\end{aligned}
$$

where the speed $v(r)$ is given by

$$
v(r)=\frac{r(1-r)}{r-2 r^{2}+\sqrt{r(2-r)}} .
$$

The speed vanishes as $v(r) \approx \sqrt{r / 2}$ as $r \rightarrow 0$. The variance of $m(n)$ grows diffusively for large $n$,

$$
\sigma_{m}^{2}=\left\langle m^{2}(n)\right\rangle-\langle m(n)\rangle^{2} \simeq D_{m}(r) n,
$$

where the diffusion coefficient (the subscript $m$ in $D_{m}(r)$ denotes the random variable $m$ ) is given by

$$
\begin{aligned}
D_{m}(r) & =\left(1-\frac{2}{\pi}\right) & & \text { for } \quad r=0, \\
& =D(r) & & \text { for } \quad r>0,
\end{aligned}
$$

where $D(r)$, for $r>0$, is given by

$$
\begin{aligned}
& D(r)=\frac{(1-r) r^{2}}{\sqrt{r(2-r)}\left[r-2 r^{2}+\sqrt{r(2-r)}\right]^{3}} \\
& \times\left[\left(2-2 r-5 r^{2}+3 r^{3}\right)+\left(2-r-r^{2}+2 r^{3}\right) \sqrt{r(2-r)}\right] .
\end{aligned}
$$

The behavior of $v(r)$ and $D(r)$, vs. $r$, are shown in Fig. 2 Note that as $r \rightarrow 0, D(r) \rightarrow 1 / 2 \neq D_{m}(0)=(1-2 / \pi)$. Thus, there is a discontinuity in the variance as $r \rightarrow 0$. These results clearly indicate that $r=0$ is a singular/critical point. Indeed, we find that near the critical point $r=0$, there is a scaling regime. Taking $r \rightarrow 0, n \rightarrow \infty$, but keeping the product $r n$ fixed, we find that the mean and the variance exhibit the following scaling behavior

$$
\begin{aligned}
\langle m(n)\rangle & \rightarrow \sqrt{n} f_{m}(r n), \\
\sigma_{m}^{2} & \rightarrow n F_{m}(r n),
\end{aligned}
$$

where the two scaling functions $f_{m}(y)$ and $F_{m}(y)$ have nontrivial expressions

$$
\begin{aligned}
& f_{m}(y)=\frac{1}{\sqrt{2 y}}\left[\left(y+\frac{1}{2}\right) \operatorname{erf}(\sqrt{y})+\sqrt{\frac{y}{\pi}} e^{-y}\right], \\
& F_{m}(y)=1+\frac{y}{2}-f_{m}^{2}(y),
\end{aligned}
$$

where $\operatorname{erf}(z)=\frac{2}{\sqrt{\pi}} \int_{0}^{z} e^{-u^{2}} d u$, is the error function. The scaling function $f_{m}(y)$ have the asymptotic behaviors: $f_{m}(y) \sim$ $\sqrt{2 / \pi}+\sqrt{2 / \pi}(y / 3)$ as $y \rightarrow 0$, and $f_{m}(y) \sim \sqrt{y / 2}+1 / \sqrt{8 y}$ as $y \rightarrow \infty$. Consequently, $F_{m}(y) \rightarrow(1-2 / \pi)$ as $y \rightarrow 0$, and $F_{m}(y) \rightarrow 1 / 2$ as $y \rightarrow \infty$. These two limiting behaviors of the scaling functions (for the mean and the variance) then smoothly interpolate between $r=0$ (strictly) and $r>0$ (and $n \rightarrow \infty$ ). For any small but fixed $r$, there is a crossover time 


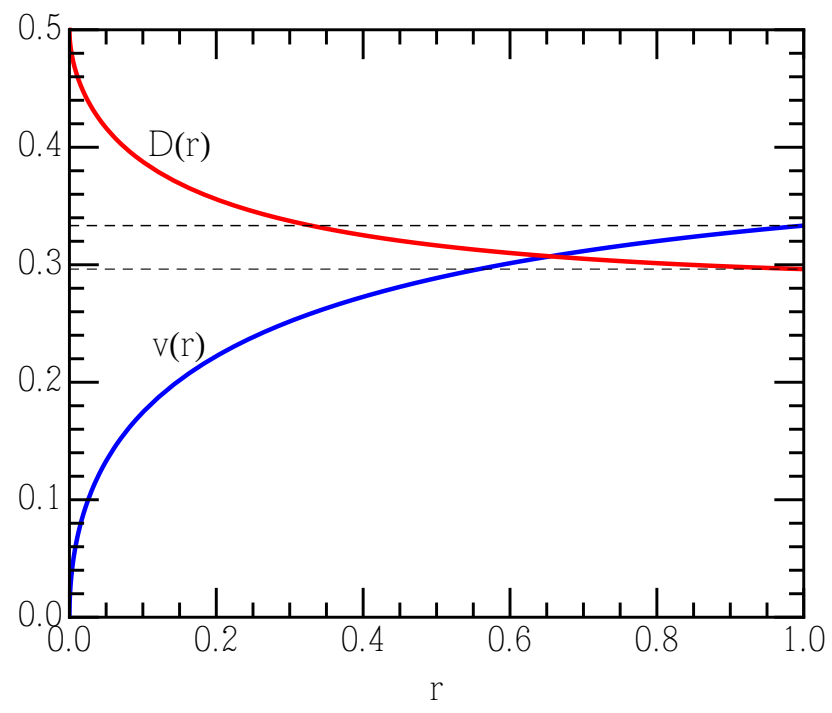

FIG. 2. (Color online) $v(r)$ and $D(r)$ as a function of $r$ (in blue and red respectively). The dashed lines show their limiting values, $1 / 3$ and $8 / 27$ respectively, for $r \rightarrow 1$.

$n^{*}(r) \sim 1 / r$, such that for $n<n^{*}(r)$, the mean and the variance grow with $n$ simply as a random walk $(r=0):\langle m(n)\rangle \sim$ $\sqrt{2 n / \pi}$ and $\left\langle\sigma_{m}^{2}\right\rangle \sim(1-2 / \pi) n$. However, for $n>n^{*}(r)$, the walker starts sensing the presence of a finite resetting rate $r$ and crosses over to a new behavior where the mean maximum grows linearly with $n,\langle m(n)\rangle \sim v(r) n$ and the variance grows diffusively, $\sigma_{m}^{2} \sim D(r) n$ with the diffusion constant $D(r)$ given in Eq. 10 .

Statistics of the position $x(n)$ : We find that the mean position behaves as

$$
\begin{aligned}
\langle x(n)\rangle & =0 & & \text { for } \quad r=0, \\
& \simeq v(r) n & & \text { for } \quad r>0,
\end{aligned}
$$

where the speed $v(r)$, for $r>0$, is the same as that of the maximum given in Eq. (6). The variance of $x(n)$ grows diffusively for large $n$,

$$
\sigma_{x}^{2}=\left\langle x^{2}(n)\right\rangle-\langle x(n)\rangle^{2} \simeq D_{x}(r) n,
$$

with the diffusion coefficient (the subscript $x$ denotes the random variable $x$ )

$$
\begin{aligned}
& D_{x}(r)=1 \quad \text { for } \quad r=0, \\
& =D(r) \quad \text { for } \quad r>0 \text {, }
\end{aligned}
$$

where the diffusion coefficient $D(r)$ is the same as that of the maximum and is given in Eq. 10p. As in the case of the maximum, the diffusion coefficient $D(r \rightarrow 0)=1 / 2 \neq D_{x}(0)=1$ undergoes a discontinuous jump at the critical point $r=0$. Similar to $m(n)$, the behavior of the mean and the variance of $x(n)$ exhibit a scaling behavior in the scaling regime $r \rightarrow 0$, $n \rightarrow \infty$ with the product $r n$ fixed

$$
\begin{aligned}
\langle x(n)\rangle & \rightarrow \sqrt{n} f_{x}(r n), \\
\sigma_{x}^{2} & \rightarrow n F_{x}(r n) .
\end{aligned}
$$

The scaling functions have the exact expressions

$$
\begin{aligned}
f_{x}(y) & =\frac{1}{\sqrt{2 y}}\left[\left(y-\frac{1}{2}\right) \operatorname{erf}(\sqrt{y})+\sqrt{\frac{y}{\pi}} e^{-y}\right], \\
F_{x}(y) & =\frac{y}{2}+\frac{1-e^{-y}}{y}-f_{x}^{2}(y) .
\end{aligned}
$$

The scaling function $f_{x}(y)$ has the asymptotic behavior: $f_{x}(y) \sim(2 / 3) \sqrt{2 / \pi} y-(2 / 15) \sqrt{2 / \pi} y^{2}$ as $y \rightarrow 0$ and $f_{x}(y) \sim \sqrt{y / 2}-1 / \sqrt{8 y}$ as $y \rightarrow \infty$. As a result, $F_{x}(y) \rightarrow 1$ as $y \rightarrow 0$ and $F_{x}(y) \rightarrow 1 / 2$ as $y \rightarrow \infty$. These scaling functions then smoothly interpolate between the critical $(r=0)$ and off-critical $(r>0)$ growth of the mean and the variance.

Statistics of the difference $y(n)=m(n)-x(n)$ : We show that the probability $Q_{\mathrm{y}}(y, n)$ that the position at the $n$-th step is at a distance $y$ away from the global maximum, has the large deviation form

$$
Q_{\mathrm{y}}(y=w n, n) \sim \exp [-n H(w)],
$$

where the large deviation function is given by

$$
H(w)= \begin{cases}w \ln \left[\frac{1+\sqrt{r(2-r)}}{1-r}\right] & \text { for } w<w^{*}, \\ \frac{w}{2} \ln \frac{1+w}{1-w}+\ln \frac{\sqrt{1-w^{2}}}{1-r} & \text { for } w>w^{*}\end{cases}
$$

with $w^{*}=\sqrt{r(2-r)}$. This result shows that for a given $n$, the probability $Q_{\mathrm{y}}(y, n)$ becomes independent of $n$ for $y<w^{*} n$ :

$$
Q_{\mathrm{y}}(y, n) \sim \exp \left[-\ln \left(\frac{1+\sqrt{r(2-r)}}{1-r}\right) y\right],
$$

while for $y>w^{*} n$, the distribution $Q_{\mathrm{y}}(y, n)$ is still timedependent. In other words, the distribution of $y$ becomes stationary on a larger and larger length scale $y^{*}(n)=w^{*} n$ that grows linearly with time $n$. Moreover, the rate function $H(w)$ is weakly non-analytic at $w=w^{*}$ : the second derivative $H^{\prime \prime}(w)$ is discontinuous at $w=w^{*}$. This signals a dynamical phase transition, similar to the one observed in the temporal evolution of the distribution of position of a Brownian motion with resetting to its initial position [11].

\section{THE DERIVATION USING GENERATING FUNCTIONS}

In this section we outline the derivation of our results. We start with the dynamics of the two basic observables $x(n)$ and $m(n)$ given in Eqs. (2) and (3). Since, we have $m \geq 0$ and $x \leq m$, it is convenient to define the difference variable $y=m-x$, where $y \geq 0$. In terms of $y$, the dynamics in Eqs. (2) and (3) get translated into the equivalent forms [see Fig.1 (b)]: 
if $y>0$

$$
(y, m) \rightarrow \begin{cases}(y-1, m) & \text { with probability }(1-r) / 2, \\ (y+1, m) & \text { with probability }(1-r) / 2, \\ (0, m) & \text { with probability } r\end{cases}
$$

and if $y=0$

$$
(y, m) \rightarrow \begin{cases}(0, m+1) & \text { with probability } 1 / 2 \\ (1, m) & \text { with probability } 1 / 2\end{cases}
$$

Let $P(x, m, n)$ and $Q(y, m, n)$ denote the joint probability distribution of $(x, m)$ and $(y, m)$ respectively, at the $n$-th time step. Evidently, $P(x, m, n)=Q(m-x, m, n)$. Using the dynamics in Eqs. (27) and (28), it is easy to write down the master equation for $Q(y, m, n)$ as

$$
\begin{array}{r}
Q(y, m, n)=\left[\frac{1-r}{2}+\right. \\
\left.+\frac{r}{2} \delta_{y, 1}\right] Q(y-1, m, n-1) \\
+\frac{1-r}{2} Q(y+1, m, n-1),
\end{array}
$$

for $y>0$, and

$$
\begin{aligned}
Q(0, m, n)=\frac{1-r}{2} Q(1, m, n-1)+ & \frac{1}{2} Q(0, m-1, n-1) \\
& +r \sum_{y=1}^{\infty} Q(y, m, n-1),
\end{aligned}
$$

with the initial condition $Q(y, m, 0)=\delta_{y, 0} \delta_{m, 0}$, and the boundary conditions $Q(y \rightarrow \infty, m, n)=0$ and $Q(y, m \rightarrow \infty, n)=0$.

To solve the set of linear equations (29) and (30), it is natural to define the generating function

$$
G(s, z, \lambda)=\sum_{y=0}^{\infty} \sum_{m=0}^{\infty} \sum_{n=0}^{\infty} Q(y, m, n) s^{y} z^{m} \lambda^{n} .
$$

Evidently, $G(1, z, \lambda)$ is the generating function for the probability distribution of the global maximum position and $G(s, 1, \lambda)$ is the generating function for the probability distribution of the position. Moreover, $G(1,1, \lambda)$ must be equal to $(1-\lambda)^{-1}$ as demanded by the normalization of the probability. After straightforward algebra, it follows that $G(s, z, \lambda)$ satisfies

$$
\begin{aligned}
& G(s, z, \lambda)\left[1-\frac{a}{2}\left(s+\frac{1}{s}\right)\right]= \\
& 1+\left(r s-\frac{1-r}{s}+z-2 r\right) \frac{\lambda}{2} F(z, \lambda)+r \lambda G(1, z, \lambda),
\end{aligned}
$$

where

$$
a=(1-r) \lambda
$$

and

$$
F(z, \lambda)=\sum_{n=0}^{\infty} \sum_{m=0}^{\infty} Q(0, m, n) z^{m} \lambda^{n}
$$

The expression for $G(1, z, \lambda)$ can be obtained by setting $s=1$ in Eq. 32 as

$$
G(1, z, \lambda)=\frac{1}{1-\lambda}\left[1-(1-z) \frac{\lambda}{2} F(z, \lambda)\right] .
$$

The normalization condition $G(1,1, \lambda)=(1-\lambda)^{-1}$ is immediately checked from above. Substituting $G(1, z, \lambda)$ in Eq. 32, after some algebra, we get

$$
\begin{aligned}
G(s, z, \lambda) & \frac{a}{2 s}\left(s_{+}-s\right)\left(s-s_{-}\right)= \\
& \frac{1-a}{1-\lambda}\left(1-\left[z_{1}(s)-z\right] \frac{\lambda}{2} F(z, \lambda)\right),
\end{aligned}
$$

where

$$
s_{ \pm}=\frac{1 \pm \sqrt{1-a^{2}}}{a}
$$

and

$$
z_{1}(s)=\frac{1-\lambda}{1-a}\left[-r s+\frac{1-r}{s}+2 r+\frac{r \lambda}{1-\lambda}\right] .
$$

In Eq. (36), the function $F(z, \lambda)$ is still undetermined and has to be determined self-consistently. To proceed, we note from Eq. (36) that $G(s, z, \lambda)$ has two poles at $s=s_{ \pm}$respectively. Therefore, inverting with respect to $s$, gives the form

$$
\sum_{\lambda=0}^{\infty} \sum_{m=0}^{\infty} Q(y, m, n) z^{m} \lambda^{n}=\frac{A}{s_{+}^{y}}+\frac{B}{s_{-}^{y}},
$$

where $A$ and $B$ are the residues at the two poles. However, from Eq. 37), we notice that $s_{+}>1$ and $s_{-}<1$. Hence, the $\left(1 / s_{-}\right)^{y}$ term in the above expression diverges when $y \rightarrow$ $\infty$, which is inconsistent with the boundary condition $Q(y \rightarrow$ $\infty, m, n)=0$. The only way to prevent this blow up is that $B$ must necessarily vanish, which implies that the right hand side of Eq. (36) vanishes for $s=s_{-}$. We note that this method of determining the self-consistency condition via the 'polecancelling' mechanism was used before in other contexts [2729]. This condition then determines $F(z, \lambda)$ as

$$
\frac{\lambda}{2} F(z, \lambda)=\frac{1}{z_{0}-z}, \quad \text { where } z_{0}=z_{1}\left(s_{-}\right) .
$$

Substituting $F(z, \lambda)$ in Eq. 35, gives the generating function of the maximum as

$$
\sum_{m=0}^{\infty} \sum_{n=0}^{\infty} P_{\mathrm{m}}(m, n) z^{m} \lambda^{n}=G(1, z, \lambda)=\frac{1}{1-\lambda} \frac{z_{0}-1}{z_{0}-z} .
$$

The normalization condition $G(1,1, \lambda)=(1-\lambda)^{-1}$ is immediately checked. It is easy to invert $G(1, z, \lambda)$ with respect to $z$ exactly, which gives

$$
\sum_{n=0}^{\infty} P_{\mathrm{m}}(m, n) \lambda^{n}=\frac{z_{0}-1}{1-\lambda} \frac{1}{z_{0}^{m+1}},
$$

where $P_{\mathrm{m}}(m, n)$ is the probability of having the maximum at $m$ in $n$ steps. 
On the other hand, substituting $F(z, \lambda)$ in Eq. 36 yields the full generating function as

$$
G(s, z, \lambda)=\frac{2 s_{+}}{a} \frac{1-r\left(1-s s_{-}\right)}{\left(s_{+}-s\right)\left(z_{0}-z\right)},
$$

where we have used $s_{+} s_{-}=1$ and

$$
z_{0}-z_{1}(s)=\left(s-s_{-}\right) s^{-1} s_{+}\left[1-r\left(1-s s_{-}\right)\right](1-\lambda)(1-a)^{-1} \text {. }
$$

Using $z_{0}=z_{1}\left(s_{-}\right)$, it can be shown that

$$
\left(z_{0}-1\right)\left(1-s_{-}\right)=2 a^{-1}(1-\lambda)\left[1-r\left(1-s_{-}\right)\right] .
$$

Therefore, the normalization condition $G(1,1, \lambda)=(1-\lambda)^{-1}$ is checked from the above expression. Finally, it is useful to rewrite the above expression as

$$
G(s, z, \lambda)=\frac{2}{a}\left[\frac{s_{+}}{s_{+}-s}-r\right] \frac{1}{z_{0}-z},
$$

where we recall that Eqs. 33, 37) and

$$
z_{0}=\frac{(1-\lambda)}{(1-a)}\left[-r s_{-}+\frac{(1-r)}{s_{-}}+2 r+\frac{r \lambda}{(1-\lambda)}\right],
$$

using Eq. 387 in $z_{0}=z_{1}\left(s_{-}\right)$. The explicit expression of the generating function $G(s, z, \lambda)$ in Eq. 45 is the central result of this paper and is valid for arbitrary $0 \leq r \leq 1$.

Let us first check a few immediate consequences of this result in Eq. 45. One can easily invert Eq. (45) with respect to $s$ and $z$ to give

$$
\sum_{n=0}^{\infty} Q(y, m, n) \lambda^{n}=\frac{2}{a}\left[s_{+}^{-y}-r \delta_{y, 0}\right] z_{0}^{-(m+1)} .
$$

Now, using $P(x, m, n)=Q(m-x, m, n)$ we get

$$
\sum_{n=0}^{\infty} P(x, m, n) \lambda^{n}=\frac{2}{a z_{0}}\left[s_{+}^{x}\left(s_{+} z_{0}\right)^{-m}-r \delta_{x, m} z_{0}^{-m}\right] .
$$

From Eq. (48), one can derive the marginal distribution of the position by summing over $m$. Note that for positive $x$, the sum over $m$ goes from $x$ to $\infty$. In contrast, for negative $x$, the sum goes from zero to $\infty$. This yields

$$
\begin{aligned}
\sum_{n=0}^{\infty} P_{\mathrm{x}}(x, n) \lambda^{n} & =\frac{2}{a\left(s_{+} z_{0}-1\right)} \\
& \times \begin{cases}{\left[(1-r) s_{+} z_{0}+r\right] z_{0}^{-(x+1)}} & \text { for } x \geq 0, \\
s_{+}^{(x+1)} & \text { for } x<0 .\end{cases}
\end{aligned}
$$

For completeness, we also compute the generating function with respect to both $x$ and $n$, which gives

$$
\sum_{x=-\infty}^{\infty} \sum_{n=0}^{\infty} P_{\mathrm{x}}(x, n) s^{x} \lambda^{n}=\frac{2}{a} \frac{s-r\left(s-s_{-}\right)}{\left(z_{0}-s\right)\left(s-s_{-}\right)} .
$$

Setting $s=1$ above and using Eq. (44), it is easy to verify the normalization condition

$$
\sum_{x=-\infty}^{\infty} \sum_{n=0}^{\infty} P_{\mathrm{x}}(x, n) \lambda^{n}=(1-\lambda)^{-1} .
$$

As mentioned above, the expression Eq. (45) for the full generating function $G(s, z, \lambda)$, valid for arbitrary reset probability $0 \leq r \leq 1$, is the main central result of our paper. Several asymptotic results for the statistics of the two random variables $m$ and $x$ can then be derived by analyzing this expression in Eq. (45) in different limits, which we present in the next few sections.

\section{THE $r=0$ CASE}

Let us first check the case without resetting, i.e., we set $r=0$. This is the standard one dimensional random walk and the results for the maximum and the position of the walker are well known. However, we reproduce it here as a check as well as for the sake of completeness.

For $r=0, a=\lambda$, and

$$
z_{0}=s_{+}=\frac{1+\sqrt{1-\lambda^{2}}}{\lambda} .
$$

Putting $\lambda=e^{-p}$, and taking $p \rightarrow 0$ limit, we have $z_{0} \sim$ $1+\sqrt{2 p},\left(z_{0}-1\right) /(1-\lambda) \sim \sqrt{(2 / p)}$ and $z_{0}^{m} \rightarrow \exp (m \sqrt{2 p})$ as $m \rightarrow \infty$ keeping $m \sqrt{p}$ fixed. Therefore, in this limit, from Eq. 42

$$
\sum_{n} P_{\mathrm{m}}(m, n) e^{-p n} \sim \frac{\sqrt{2}}{\sqrt{p}} \exp (-m \sqrt{2 p}) .
$$

Inverting the Laplace transform gives,

$$
P_{\mathrm{m}}(m, n) \sim \sqrt{\frac{2}{\pi n}} \exp \left(-\frac{m^{2}}{2 n}\right), \quad \text { where } m \geq 0 .
$$

From this distribution, it is easy to compute all the moments for large $n$. For instance, the mean and the variance of the maximum grow asymptotically as

$$
\langle m(n)\rangle \simeq \sqrt{\frac{2 n}{\pi}} \quad \text { and } \quad \sigma_{m}^{2} \simeq\left(1-\frac{2}{\pi}\right) n,
$$

as stated respectively in Eqs. (4) and (8).

Similarly, from Eq. 49], we get,

$$
\sum_{n} P_{\mathrm{X}}(x, n) e^{-p n} \sim \frac{1}{\sqrt{2 p}} \exp (-|x| \sqrt{2 p}),
$$

which, after Laplace inversion, gives the expected Gaussian distribution (since a random walk, for large $n$, converges to a Brownian motion)

$$
P_{\mathrm{X}}(x, n) \sim \frac{1}{\sqrt{2 \pi n}} \exp \left(-\frac{x^{2}}{2 n}\right) .
$$

Thus the mean and the variance of the position behave as

$$
\langle x(n)\rangle=0 \quad \text { and } \quad \sigma_{x}^{2} \simeq n,
$$

as stated respectively in Eqs. (15) and (18). 
For the joint distribution of the maximum and the position, we get from Eq. (48)

$$
\sum_{n=0}^{\infty} P(x, m, n) e^{-p n} \sim 2 \exp [-(2 m-x) \sqrt{2 p}]
$$

which, after Laplace inversion, gives

$$
P(x, m, n) \sim \frac{(2 m-x)}{n^{3 / 2}} \sqrt{\frac{2}{\pi}} \exp \left[-\frac{(2 m-x)^{2}}{2 n}\right],
$$

where $x \leq m$ and $m \geq 0$.

Let us also verify Eq. (50) using the exact result of the random walk

$$
P_{\mathrm{x}}(x, n)=\left(\begin{array}{c}
n \\
\frac{n+x}{2}
\end{array}\right) 2^{-n}, \text { when } n+x \text { is even, }
$$

and zero otherwise. Therefore,

$$
\sum_{x=-n}^{n} P_{\mathrm{x}}(x, n) s^{x}=\sum_{m=0}^{n} P(2 m-n, n) s^{2 m-n}=\left[\frac{1}{2}\left(s+\frac{1}{s}\right)\right]^{n} .
$$

Consequently,

$$
\begin{aligned}
\sum_{n=0}^{\infty} \lambda^{n} \sum_{x=-n}^{n} P_{\mathrm{x}}(x, n) s^{x} & =\left[1-\frac{\lambda}{2}\left(s+\frac{1}{s}\right)\right]^{-1} \\
& =\frac{2 s}{\lambda} \frac{1}{\left(s_{+}-s\right)\left(s-s_{-}\right)},
\end{aligned}
$$

which is same as Eq. (50) for $r=0$.

Thus, for the case $r=0$, when there is no resetting to the maximum, we have recovered the results for the usual random walk.

\section{THE $r \rightarrow 1$ LIMIT}

In the other extreme limit of $r \rightarrow 1$, we have $z_{0}=(2-$ $\left.\lambda^{2}\right) / \lambda$. Therefore, from Eq. 42

$$
\sum_{n=0}^{\infty} P_{\mathrm{m}}(m, n) \lambda^{n}=\frac{2+\lambda}{2-\lambda^{2}}\left[\frac{\lambda}{2-\lambda^{2}}\right]^{m} .
$$

From the series expansion of the above expression with respect to $\lambda$, it is easily verified that $P_{\mathrm{m}}(m, 0)=\delta_{m, 0}$. Moreover,

$$
P_{\mathrm{m}}(0, n)=2^{-\frac{(n+1)}{2}}\left[\frac{1-(-1)^{n}}{2}+\sqrt{2} \frac{1+(-1)^{n}}{2}\right],
$$

is the probability that the walker has not crossed the origin up to the step $n$. This is the case where the walker goes to the site $x=-1$ (with probability $1 / 2$ ) and comes back to the origin (with probability one) at alternate time steps. In general (for $r \rightarrow 1)$,

$$
P_{\mathrm{m}}(m, n)=\frac{1}{2 \pi i} \oint_{0} \frac{d \lambda}{\lambda^{n+1}} \frac{2+\lambda}{2-\lambda^{2}}\left[\frac{\lambda}{2-\lambda^{2}}\right]^{m} .
$$

For large $m$ and $n$, with $m=w n$, the integral can be evaluated using saddle-point approximation, which to the leading order gives

$$
P_{\mathrm{m}}(m=w n, n) \sim \exp [-n S(w)]
$$

where $S(w) \equiv S\left(w, \lambda^{*}\right)$ with

$$
S(w, \lambda)=\ln \lambda-w \ln \left[\frac{\lambda}{2-\lambda^{2}}\right] .
$$

The saddle point $\lambda^{*}$ is obtained by solving the equation $\left.\partial_{\lambda} S(w, \lambda)\right|_{\lambda^{*}}=0$, as $\left[\lambda^{*}(w)\right]^{2}=2(1-w) /(1+w)$. Substituting this in the above equation we obtain the large deviation function as

$$
S(w) \equiv S\left(w, \lambda^{*}\right)=\frac{(1-w)}{2} \ln \frac{2(1-w)}{(1+w)}+w \ln \frac{4 w}{(1+w)} .
$$

This large deviation function has a maximum at $w=1 / 3$, and near this, one gets $S(w)=(27 / 16)(w-1 / 3)^{2}$, which implies the Gaussian form

$$
P_{\mathrm{m}}(m, n) \sim \exp \left(-\frac{(m-n / 3)^{2}}{2 D n}\right), \text { with } D=\frac{8}{27} .
$$

In fact, Eq. 63) can be inverted exactly, which gives

$$
P_{\mathrm{m}}(m, n)= \begin{cases}2^{-\frac{(n+m)}{2}}\left(\begin{array}{c}
\frac{n+m}{2} \\
m
\end{array}\right) & \text { if }(n-m) \text { is even, } \\
2^{-\frac{(n+m+1)}{2}\left(\begin{array}{c}
\frac{n+m-1}{2} \\
m
\end{array}\right)} & \text { if }(n-m) \text { is odd. }\end{cases}
$$

For large $n$ and $m$, using the Stirling's approximation in Eq. (70), one can recover the large deviation function given by Eq. 68).

Let us now look at the probability distribution of the position using Eq. (49). For $r \rightarrow 1$ we have $a \rightarrow 0$ and $s_{+} \rightarrow \infty$ with $a s_{+} \rightarrow 2$. Therefore, from Eq. (49), it is clear that for negative $x$, we get nonzero probability only for $x=-1$, which reads

$$
\sum_{n=0}^{\infty} P_{\mathrm{x}}(-1, n) \lambda^{n}=\frac{1}{z_{0}}=\frac{\lambda}{2-\lambda^{2}}
$$

Inverting this with respect to $\lambda$ gives

$$
P_{\mathrm{X}}(-1, n)=\frac{1-(-1)^{n}}{2} 2^{-\frac{n+1}{2}} .
$$

This result can be understood, as this is the case where the walker goes to the site $x=-1$ (with probability $1 / 2$ ) at odd time steps and comes back to the origin (with probability one) at even time steps. The also implies that $P(-1, n)$ is nonzero only when the maximum remains zero. Indeed from Eq. (48, for $r \rightarrow 1$ we get $\sum_{n} P(-1,0, n) \lambda^{n}=z_{0}^{-1}=\sum_{n} P_{\mathrm{x}}(-1, n) \overline{\lambda^{n}}$. For $x \geq 0$, Eq. 49) we get

$$
\sum_{n=0}^{\infty} P_{\mathrm{x}}(x, n) \lambda^{n}=\frac{4-\lambda^{2}}{\left(2-\lambda^{2}\right)^{2}}\left[\frac{\lambda}{2-\lambda^{2}}\right]^{x}
$$


Although the exact form differs from Eq. 63, it is evident that $P_{\mathrm{x}}(x, n)$ and $P_{\mathrm{m}}(m, n)$ have the same large deviation function, i.e.,

$$
P_{\mathrm{X}}(x=w n, n) \sim \exp [-n S(w)]
$$

with $S(w)$ given by Eq. 68.

\section{THE CASE $0<r<1$}

For general resetting probability $0<r<1$, it is a bit cumbersome to find the exact large deviation functions associated with the probabilities $P_{\mathrm{m}}(m, n)$ and $P_{\mathrm{x}}(x, n)$. However, one expects (as in the cases of $r=0,1)$ the typical fluctuations near the mean to be governed by Gaussian distributions. The generating functions for the mean and the variance for the maximum and the current position are obtained from the generating functions of their distributions, given by Eq. (41) and Eq. (50) respectively, simply by taking derivatives. From their respective generating functions, we then derive, for arbitrary but fixed $0<r<1$, the asymptotic behavior of the mean and variance for large $n$ for both the maximum and the position. Finally, the asymptotic behavior of the the full probability distribution of the difference variable $y(n)=m(n)-x(n)$ is derived. These derivations are outlined in the next three subsections.

\section{A. Statistics of the maximum $m(n)$}

From Eq. 41, we get the generating functions of the first two moments of the maximum as

$$
\sum_{n=0}^{\infty}\langle m(n)\rangle \lambda^{n}=\left.\frac{\partial}{\partial z} G(1, z, \lambda)\right|_{z=1}=\frac{1}{1-\lambda} \frac{1}{z_{0}-1},
$$

and

$$
\begin{aligned}
\sum_{n=0}^{\infty}\left\langle m^{2}(n)\right\rangle \lambda^{n} & =\left.\frac{\partial}{\partial z} z \frac{\partial}{\partial z} G(1, z, \lambda)\right|_{z=1} \\
& =\frac{1}{1-\lambda}\left[\frac{2}{\left(z_{0}-1\right)^{2}}+\frac{1}{z_{0}-1}\right]
\end{aligned}
$$

respectively. Using the expression of $z_{0}=z_{1}\left(s_{-}\right)$from Eq. (38, it is easy to check that

$$
\frac{1}{z_{0}-1}=\frac{C(r, \lambda)}{1-\lambda}
$$

where

$$
C(r, \lambda)=\frac{a(1-a)}{(1-a)(1-2 r)+\sqrt{1-a^{2}}},
$$

with $a=(1-r) \lambda$. Inverting Eqs. (75) and 76 with respect to $\lambda$ using Cauchy's formula, we get

$$
\langle m(n)\rangle=\frac{1}{2 \pi i} \oint_{0} \frac{d \lambda}{\lambda^{n+1}} \frac{C(r, \lambda)}{(1-\lambda)^{2}}
$$

and

$$
\left\langle m^{2}(n)\right\rangle=\frac{1}{2 \pi i} \oint_{0} \frac{d \lambda}{\lambda^{n+1}}\left[\frac{2 C^{2}(r, \lambda)}{(1-\lambda)^{3}}+\frac{C(r, \lambda)}{(1-\lambda)^{2}}\right]
$$

respectively, where the integral is along a counterclockwise closed contour around the origin in the complex $\lambda$ plane.

A hint that $r=0$ is a special case and is different from the $r>0$ case can be already seen at this level. For $r=0, a=\lambda$ and from Eq. 78 one gets $C(0, \lambda)=(1-\lambda) \lambda[(1-\lambda)+$ $\left.\sqrt{1-\lambda^{2}}\right]^{-1}$. Therefore, for the $r=0$ case, the powers of $(1-$ $\lambda)$ in the denominators of the above expressions, reduce by one, and hence this case must be treated separately from the $r>0$ case. In the following we will only consider the case $r>0$.

Evaluating the contour integrals in Eqs. 79 and 80 explicitly for all $n$ looks cumbersome. However, the asymptotic behavior for large $n$ can be derived by separating out the contributions to the contour integrals arising from the pole at $\lambda=1$. Note that $C(r, \lambda)$ also has two branch points at $\lambda= \pm(1-r)^{-1}$. Therefore, $\oint_{0}=-\oint_{1}+$ [contributions from the integrals around the branch cuts from $-\infty$ to $-(1-r)^{-1}$ and from $(1-r)^{-1}$ to $\infty$ ]. Using the residue theorem and computing the residue at $\lambda=1$ we get

$$
\begin{aligned}
\frac{1}{2 \pi i} \oint_{0} \frac{d \lambda}{\lambda^{n+1}} \frac{C(r, \lambda)}{(1-\lambda)^{2}} & =C_{0}(r)(n+1)+C_{1}(r) \\
+ & {[\text { branch cuts contributions] }}
\end{aligned}
$$

and

$$
\begin{aligned}
\frac{1}{2 \pi i} \oint_{0} \frac{d \lambda}{\lambda^{n+1}} \frac{2 C^{2}(r, \lambda)}{(1-\lambda)^{3}}=(n+1)(n+2) C_{0}^{2}(r) \\
+4(n+1) C_{0}(r) C_{1}(r)+2 C_{1}^{2}(r)+2 C_{0}(r) C_{2}(r) \\
+[\text { branch cuts contributions], }
\end{aligned}
$$

where

$$
C_{n}(r)=\left.(-1)^{n} \frac{\partial^{n}}{\partial \lambda^{n}} C(r, \lambda)\right|_{\lambda=1}=\left.\frac{\partial^{n}}{\partial \lambda^{n}} C(r, 1-\lambda)\right|_{\lambda=0} .
$$

These coefficients can be calculated explicitly using Eq. (78). For example, the first two coefficients are given explicitly as

$$
C_{0}(r)=\frac{r(1-r)}{r(1-2 r)+\sqrt{2 r-r^{2}}}
$$

and

$$
C_{1}(r)=\frac{r(1-r)\left[1-3 r+r^{2}+r(2 r-1) \sqrt{2 r-r^{2}}\right]}{\sqrt{2 r-r^{2}}\left(r-2 r^{2}+\sqrt{2 r-r^{2}}\right)^{2}} .
$$

One can also show that the branch cuts contributions in Eqs. (81) and 82 go to zero exponentially fast as $n \rightarrow \infty$.

Using the results from Eqs. 81) and (82) in Eqs. (79) and $(80)$, we then obtain, for large $n$, the mean

$$
\langle m(n)\rangle \simeq v(r) n, \text { with } v(r)=C_{0}(r)=\frac{r(1-r)}{r(1-2 r)+\sqrt{2 r-r^{2}}} .
$$


The speed $v(r)$, as a function of $r$, is plotted in Fig. 2 Similarly, the variance grows linearly for large $n$

$$
\sigma_{m}^{2}=\left\langle m^{2}\right\rangle-\langle m\rangle^{2} \simeq D_{m}(r) n,
$$

with

$$
D_{m}(r)=D(r) \equiv C_{0}^{2}(r)+2 C_{0}(r) C_{1}(r)+C_{0}(r) .
$$

Using $C_{0}(r)$ and $C_{1}(r)$ from Eqs. 84 and 85 gives the explicit expression for the diffusion coefficient $D(r)$, for $r>0$, as given by Eq. 10). A plot of $D(r)$ vs. $r$ is provided in Fig.2

\section{B. Statistics of the position $x(n)$}

Similarly, to compute the mean and variance of the position $x(n)$, we take derivatives with respect to $s$ in Eq. 50) at $s=1$ to get

$$
\sum_{n=0}^{\infty}\langle x(n)\rangle \lambda^{n}=\frac{2}{a}\left[\frac{1-r\left(1-s_{-}\right)}{\left(1-s_{-}\right)\left(z_{0}-1\right)^{2}}-\frac{s_{-}}{\left(1-s_{-}\right)^{2}\left(z_{0}-1\right)}\right]
$$

and

$$
\begin{aligned}
\sum_{n=0}^{\infty}\left\langle x^{2}(n)\right\rangle \lambda^{n}=\frac{2}{a}\left[\frac{2\left[1-r\left(1-s_{-}\right)\right]}{\left(1-s_{-}\right)\left(z_{0}-1\right)^{3}}\right. & \\
& \left.+\frac{1-r\left(1-s_{-}\right)^{2}-3 s_{-}}{\left(1-s_{-}\right)^{2}\left(z_{0}-1\right)^{2}}+\frac{s_{-}\left(1+s_{-}\right)}{\left(1-s_{-}\right)^{3}\left(z_{0}-1\right)}\right],
\end{aligned}
$$

where we recall Eqs. 33, , 37) and (46).

It is useful to define the following quantities

$$
\begin{aligned}
& A_{1}(r, \lambda)=\frac{2\left[1-r\left(1-s_{-}\right)\right]}{a\left(1-s_{-}\right)}, \\
& A_{2}(r, \lambda)=\frac{2 s_{-}}{a\left(1-s_{-}\right)^{2}}, \\
& A_{3}(r, \lambda)=\frac{2\left[1-r\left(1-s_{-}\right)^{2}-3 s_{-}\right]}{a\left(1-s_{-}\right)^{2}}, \\
& A_{4}(r, \lambda)=\frac{2 s_{-}\left(1+s_{-}\right)}{a\left(1-s_{-}\right)^{3}} .
\end{aligned}
$$

We note that $A_{1}(r, \lambda) C(r, \lambda)=1$ and $\left[2 A_{2}(r, \lambda)+\right.$ $\left.A_{3}(r, \lambda)\right] C(r, \lambda)=1$. Using these definitions and Eq. (77), we invert the above generating function with respect to $\lambda$ and get

$$
\begin{array}{r}
\langle x(n)\rangle=\frac{1}{2 \pi i} \oint_{0} \frac{d \lambda}{\lambda^{n+1}}\left[\frac{C(r, \lambda)}{(1-\lambda)^{2}}-\frac{A_{2}(r, \lambda) C(r, \lambda)}{(1-\lambda)}\right], \\
\left\langle x^{2}(n)\right\rangle=\frac{1}{2 \pi i} \oint_{0} \frac{d \lambda}{\lambda^{n+1}}\left[\frac{2 C^{2}(r, \lambda)}{(1-\lambda)^{3}}+\frac{A_{3}(r, \lambda) C^{2}(r, \lambda)}{(1-\lambda)^{2}}\right. \\
\left.+\frac{A_{4}(r, \lambda) C(r, \lambda)}{(1-\lambda)}\right] .
\end{array}
$$

Finally, evaluating these integrals using residue theorem, we get

$$
\langle x(n)\rangle=(n+1) C_{0}(r)+C_{1}(r)-A_{2,0}(r) C_{0}(r)+\ldots,
$$

and

$$
\begin{aligned}
\left\langle x^{2}(n)\right\rangle & =(n+1)(n+2) C_{0}^{2} \\
& +(n+1)\left[4 C_{0}(r) C_{1}(r)+A_{3,0}(r) C_{0}^{2}(r)\right] \\
& +2 C_{1}^{2}(r)+2 C_{0}(r) C_{2}(r)+A_{3,1}(r) C_{0}^{2}(r) \\
& +2 A_{3,0}(r) C_{0}(r) C_{1}(r)+A_{4,0}(r) C_{0}(r)+\ldots,
\end{aligned}
$$

where $C_{n}(r)$ is defined by Eq. 83 and

$$
A_{l, n}(r)=\left.(-1)^{n} \frac{\partial^{n}}{\partial \lambda^{n}} A_{l}(r, \lambda)\right|_{\lambda=1}=\left.\frac{\partial^{n}}{\partial \lambda^{n}} A_{l}(r, 1-\lambda)\right|_{\lambda=0} .
$$

Finally, collecting the leading term for large $n$, we obtain the following asymptotic results for the mean and the variance. For large $n$, and for $r>0$, the mean

$$
\langle x(n)\rangle \sim v(r) n,
$$

where the speed $v(r)=C_{0}(r)$ turns out to be exactly the same as in case of maximum, with explicit expression given in Eq. 866. Similarly, the variance behaves, asymptotically for large $n$ and for all $r>0$, as

$$
\begin{aligned}
& \sigma_{x}^{2}=\left\langle x^{2}(n)\right\rangle-\langle x(n)\rangle^{2} \simeq D_{x}(r) n, \\
& \text { with } D_{x}(r)=D(r) \equiv C_{0}^{2}(r)+2 C_{0}(r) C_{1}(r)+C_{0}(r) .
\end{aligned}
$$

Hence, the first and the second moments of the two variables $m(n)$ and $x(n)$ grow identically for large $n$. It is then natural to ask how the difference variable $y(n)=m(n)-x(n)$ is distributed and we address this in the next subsection

\section{Asymptotic distribution of the difference $y(n)=m(n)-x(n)$}

Let $Q_{\mathrm{y}}(y, n)$ be the probability that the location at the $n$-th step is at a distance $y$ away from the global maximum. Clearly, $Q_{\mathrm{y}}(y, n)=\sum_{m=0}^{\infty} Q(y, m, n)$. Therefore, from Eq. 477) we get

$$
\sum_{n=0}^{\infty} Q_{\mathrm{y}}(y, n) \lambda^{n}=\frac{2}{a}\left[s_{+}^{-y}-r \delta_{y, 0}\right] \frac{1}{z_{0}-1} .
$$

Let us first look at the value $y=0$, for which Eq. 102 reads

$$
\sum_{n=0}^{\infty} Q_{\mathrm{y}}(0, n) \lambda^{n}=\frac{2}{\lambda} \frac{1}{z_{0}-1}=\frac{2 C(r, \lambda)}{\lambda(1-\lambda)}
$$

where we have used Eq. (77). To extract the large $n$ behavior of $Q_{\mathrm{y}}(0, n)$, we need to investigate the right hand side of Eq. 103 in the limit $\lambda \rightarrow 1$. As $\lambda \rightarrow 1, C(r, \lambda) \rightarrow C(r, 1)=$ $C_{0}(r)$ given in Eq. (84). Hence, the right hand side of Eq. 103 behaves as $2 C_{0}(r) /(1-\lambda)$ as $\lambda \rightarrow 1$. This clearly indicates that $Q_{\mathrm{y}}(0, n)$ becomes independent of $n$ in the limit $n \rightarrow \infty$, given by

$$
Q_{\mathrm{y}}(0, n \rightarrow \infty) \rightarrow 2 C_{0}(r) .
$$

For $y>0$, inverting the generating function Eq. 102 we get

$$
Q_{\mathrm{y}}(y, n)=\frac{1}{2 \pi i} \oint_{0} \frac{d \lambda}{\lambda^{n+1}} \frac{2}{a\left(z_{0}-1\right)} s_{+}^{-y} .
$$


Now, using explicit expressions, and changing the integral over $\lambda$ to that over $a=(1-r) \lambda$, we get

$$
\begin{aligned}
Q_{\mathrm{y}}(y, n)=\frac{1}{2 \pi i} \oint_{0} & \frac{d a}{\left(a_{0}-a\right)}\left[\frac{2(1-a)}{(1-a)\left(2 a_{0}-1\right)+\sqrt{1-a^{2}}}\right] \\
& \times\left(\frac{a_{0}}{a}\right)^{n+1}\left[\frac{a}{1+\sqrt{1-a^{2}}}\right]^{y},
\end{aligned}
$$

where $a_{0}=1-r$. The integrand has a simple pole at $a=a_{0}$ and branch points at $a= \pm 1$. The contour of integration around zero can be split into two vertical contours: one that goes from $+i \infty$ to $-i \infty$ through the left of the origin and another that goes from $-i \infty$ to $+i \infty$ through the right of the origin. The left contour subsequently can be wrapped around the branch cut from $a=-1$ to $-\infty$. The contribution from this contour is subdominant and the main contribution comes from the contour on the right for large $n$. It is useful to express the integral as

$$
Q_{\mathrm{y}}(y=w n, n) \approx \frac{1}{2 \pi i} \int_{\left(0_{+}\right)-i \infty}^{\left(0_{+}\right)+i \infty} d a \frac{g(a)}{\left(a_{0}-a\right)} \exp [-n H(w, a)]
$$

so that for large $n$ we can use the saddle point approximation method. Here

$$
H(w, a)=\ln \left(a / a_{0}\right)-w \ln \left[\frac{a}{1+\sqrt{1-a^{2}}}\right],
$$

and

$$
g(a)=\frac{2(1-a)}{(1-a)\left(2 a_{0}-1\right)+\sqrt{1-a^{2}}} .
$$

The saddle point $a^{*}$ is obtained by solving the condition $\left.\partial_{a} H(w, a)\right|_{a^{*}}=0$, which gives $a^{*}=\sqrt{1-w^{2}}$. Note that the integrand of Eq. (107) has a simple pole at $a=a_{0}=1-r$. For $w<w^{*}=\sqrt{r(2-r)}$, we have $a^{*}>a_{0}$, and therefore, the contribution to the above integral comes from both the pole and the saddle point. However, the contribution from the pole is larger than that from the saddle point. Therefore, for large $n, Q_{\mathrm{y}}(y=w n, n) \sim \exp \left[-n H_{1}(w)\right]$, where the large deviation function is given by

$$
H_{1}(w) \equiv H\left(w, a_{0}\right)=w \ln \left[\frac{1+\sqrt{r(2-r)}}{1-r}\right] .
$$

On the other hand, for $w>w^{*}$, we have $a^{*}<a_{0}$. Therefore, evaluating the above integral using saddle-point approximation gives $Q_{\mathrm{y}}(y=w n, n) \sim \exp \left[-n H_{2}(w)\right]$ where the large deviation function is given by

$$
H_{2}(w) \equiv H\left(w, a^{*}\right)=\frac{w}{2} \ln \frac{1+w}{1-w}+\ln \frac{\sqrt{1-w^{2}}}{1-r} .
$$

Finally, combining these two regimes we obtain the large deviation behavior

with the rate function given by

$$
Q_{\mathrm{y}}(y=w n, n) \sim \exp [-n H(w)],
$$

$$
H(w)= \begin{cases}H_{1}(w)=w \ln \left[\frac{1+\sqrt{r(2-r)}}{1-r}\right] & \text { for } w<w^{*}, \\ H_{2}(w)=\frac{w}{2} \ln \frac{1+w}{1-w}+\ln \frac{\sqrt{1-w^{2}}}{1-r} & \text { for } w>w^{*},\end{cases}
$$

with $w^{*}=\sqrt{r(2-r)}$.

As discussed in Section 2, this result indicates that for a given large $n, Q_{\mathrm{y}}(y, n)$ becomes independent of $n$ for $y<w^{*} n$ and is still $n$-dependent for $y>w^{*} n$, signalling a dynamical phase transition. The rate function $H(w)$, plotted in Fig. 3 , is weakly singular at the critical point $w=w^{*}$ where both $H(w)$ and $H^{\prime}(w)$ are continuous, but the second derivative $H^{\prime \prime}(w)$ is discontinuous: $H^{\prime \prime}\left(w \rightarrow w^{*-}\right)=0$, while $H^{\prime \prime}\left(w \rightarrow w^{*+}\right)=$ $1 /(1-r)^{2}$. Such a second order dynamical phase transition was also observed recently in the time evolution of the distribution of position of a Brownian motion in one dimension with resetting to its initial position [11].

We finish this subsection by making a couple of interesting observations. In the limit $r \rightarrow 0$ we have $w^{*}=0$. Therefore, the large deviation form is given by $H_{2}(w)$ with $r=0$. Expanding in Taylor series, for small $w$ we get $H_{2}(w) \simeq w^{2} / 2$, which gives the Gaussian form $Q_{\mathrm{y}}(y, n) \sim \exp \left[-y^{2} /(2 n)\right]$. This is consistent with the result obtained by substituting $x=m-y$ in Eq. (60) and integrating over $m$ from 0 to $\infty$. On the other hand, in the limit $r \rightarrow 1$, we have $w^{*}=1$. Therefore, the large deviation form is given by $H_{1}(w)$ with $r=1$. We note that for $r=1$, the large deviation function is $-\infty$, except for the case $w=0$. This is because, $y$ can take only two values, namely, 0 and 1 , for the case $r=1$. Using the result of Eq. 104, we get $Q_{\mathrm{y}}(0, n \rightarrow \infty) \rightarrow 2 C_{0}(1)=2 / 3$. For $y=1$, Eq. (102) gives

$$
\sum_{n=0}^{\infty} Q_{\mathrm{y}}(1, n) \lambda^{n}=\frac{2}{\left(a s_{+}\right)\left(z_{0}-1\right)}
$$

Now, in the limit $r \rightarrow 1$, we get $a s_{+} \rightarrow 2$ and $\left(z_{0}-1\right) \rightarrow$ $\lambda^{-1}(1-\lambda)(2+\lambda)$. Using these, and inverting the above equation we get

$$
Q_{\mathrm{y}}(1, n)=\frac{1}{3}\left[1-\left(-\frac{1}{2}\right)^{n}\right] \rightarrow \frac{1}{3} \quad \text { as } n \rightarrow \infty .
$$

Finally, following the method used in Ref. [30] in a different context, we can also write down a more complete asymptotic form of $Q(y, n)$ for large $n$ as 
$Q_{\mathrm{y}}(y=w n, n) \approx \frac{e^{-n H_{2}(w)}}{2 \sqrt{\pi n}}\left[K(w)-\frac{\operatorname{sgn}\left(w^{*}-w\right) g_{-1}}{\sqrt{H_{2}(w)-H_{1}(w)}}\right]+e^{-n H_{1}(w)} g_{-1}\left[\frac{\operatorname{sgn}\left(w^{*}-w\right)}{2} \operatorname{erfc}\left(\sqrt{n\left[H_{2}(w)-H_{1}(w)\right]}\right)-\theta\left(w^{*}-w\right)\right]$

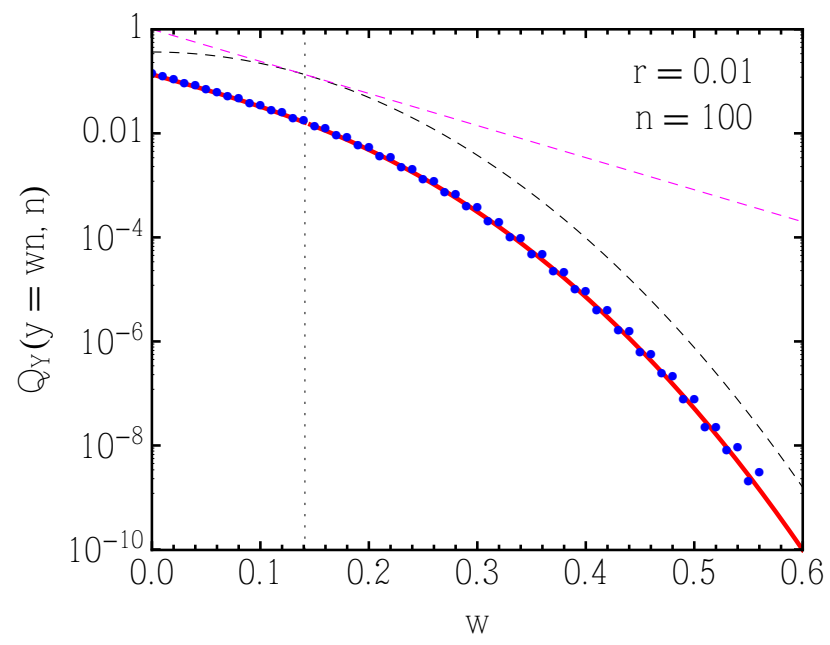

FIG. 3. (Color online) $Q(y, n)$ against the scaled variable $w=y / n$ for $n=100$ and $r=0.01$. The (blue) points are obtained from numerical simulation. The solid (red) line represents the asymptotic form given by Eq. 116. The dashed lines (magenta and black) plot the large deviation forms $Q_{\mathrm{y}}(y=w n, n) \sim \exp \left[-n H_{1,2}(w)\right]$ with the large deviation functions $H_{1}(w)$ and $H_{2}(w)$ given by Eq. 110 and Eq. (111) respectively. The vertical dotted line marks the position of $w^{*} \approx 0.141$.

where $g_{-1}=-g\left(a_{0}\right)$ and $K(w)=\sqrt{2} w g\left(a^{*}\right) /\left(a_{0}-a^{*}\right)$. This pre-asymptotic form is particularly useful to compare to the results of simulation. Indeed, Figure 3 compares this form with the numerical simulation results. The agreement is excellent.

\section{THE LIMIT $r \rightarrow 0$ AND THE ASSOCIATED SCALING FUNCTIONS}

From the results presented in Section IV for $r=0$ and in Section VI for $r>0$, we see that the limit $r \rightarrow 0$ (after taking the large $n$ limit) is not the same as $r=0$, for the statistics of both the maximum and the position. In other words, the two limits $\lim _{r \rightarrow 0}$ and $\lim _{n \rightarrow \infty}$ do not commute. This indicates that $r=0$ is a singular or a 'critical' point. For finite but large $n$, there should then be a smooth crossover function interpolating between these two limits. In this section, these crossover scalings functions are derived analytically and compared to numerical simulations.

\section{A. Scaling functions associated with the maximum $m(n)$}

We first consider the crossover scaling functions (near $r \rightarrow$ 0 ) associated with the mean and the variance of the maximum $m(n)$. Let us first focus on the mean. Our starting point is the exact generating function for the mean in Eq. (75), where we recall that $z_{0}=z_{1}\left(s_{-}\right)$is given in Eq. 38 . We also remind the reader that

$$
a=(1-r) \lambda \quad \text { and } \quad s_{-}=\frac{1}{a}\left(1-\sqrt{1-a^{2}}\right) .
$$

Since, we want to analyze the behavior of $\langle m(n)\rangle$ for large $n$, and simultaneously $r \rightarrow 0$, we need to set $\lambda$ close to 1 in Eq. 75. We set, $\lambda=1-p$ where $p$ is small. Upon inspecting $s_{-}$in Eq. (117), it follows that the right scaling limit is when $p \rightarrow 0, r \rightarrow 0$, keeping the ratio $p / r$ fixed. In the real space, this limit corresponds to $r \rightarrow 0, n \rightarrow \infty$ but keeping the product $r n$ fixed. In this limit, the leading behavior of $s_{-}$can be easily worked out to give

$$
s_{-} \approx 1-\sqrt{2(r+p)}
$$

Substituting this leading behavior of $s_{-}$in Eq. (38), it follows that in the scaling limit

$$
z_{0}=z_{1}\left(s_{-}\right) \approx 1+\frac{\sqrt{2} p}{\sqrt{r+p}}
$$

We substitute this leading behavior of $z_{0}$ on the right hand side (rhs) of Eq. 75 and use $\lambda=1-p$. In the limit $p \rightarrow 0$, the sum on the left hand side (lhs) of Eq. (75) can be approximated by an integral, yielding in the scaling limit

$$
\int_{0}^{\infty}\langle m(n)\rangle e^{-p n} d n \approx \frac{\sqrt{r+p}}{\sqrt{2} p^{2}}
$$

By power counting on both sides of Eq. (120), it follows that $\langle m(n)\rangle$ must have the following scaling behavior

$$
\langle m(n)\rangle \approx \sqrt{n} f_{m}(r n)
$$

in the appropriate scaling limit $r \rightarrow 0, n \rightarrow \infty$ with the product $r n$ fixed.

Substituting this scaling behavior on the lhs of Eq. (120), setting $p / r=s$ and making a change of variable $r n=y$, yields the following equation for the scaling function $f_{m}(y)$

$$
\int_{0}^{\infty} \sqrt{y} f_{m}(y) e^{-s y} d y=\frac{\sqrt{1+s}}{\sqrt{2} s^{2}} .
$$


To invert the Laplace transform on the rhs of Eq. 122, we reexpress

$$
\frac{\sqrt{1+s}}{s^{2}}=\frac{1}{s^{2} \sqrt{1+s}}+\frac{1}{s \sqrt{1+s}} .
$$

Each term on the rhs of Eq. (123) is an elementary function that can be easily inverted using convolution theorem. Inverting, we then get an exact expression for the scaling function

$$
f_{m}(y)=\frac{1}{\sqrt{2 y}}\left[\left(y+\frac{1}{2}\right) \operatorname{erf}(\sqrt{y})+\sqrt{\frac{y}{\pi}} e^{-y}\right],
$$

where $\operatorname{erf}(z)=\frac{2}{\sqrt{\pi}} \int_{0}^{z} e^{-u^{2}} d u$. The function $f_{m}(y)$ has the following asymptotic behaviors

$$
f_{m}(y) \sim \begin{cases}\sqrt{\frac{2}{\pi}}+O(y) & \text { as } y \rightarrow 0, \\ \sqrt{\frac{y}{2}}+O\left(\frac{1}{\sqrt{y}}\right) & \text { as } y \rightarrow \infty .\end{cases}
$$

Thus, when $r=0$, using $f_{m}(0)=\sqrt{2 / \pi}$ in Eq. $\sqrt{121}$ yields the asymptotic behavior of the mean, $\langle m(n)\rangle \simeq \sqrt{2 n / \pi}$. In contrast, when $r>0$, as $n \rightarrow \infty$, the scaling argument $y=r n \rightarrow$ $\infty$. Hence, using the other asymptotic behavior in Eq. (125) as $y \rightarrow \infty$, yields the linear growth $\langle m(n)\rangle \simeq \sqrt{r / 2} n$. Note that the speed $v(r)$ in Eq. 6 indeed tends to $v(r) \rightarrow \sqrt{r / 2}$ as $r \rightarrow 0$. The exact scaling function $f_{m}(y)$ thus interpolates smoothly between these two limits. For any small but nonzero $r$, we thus expect that $\langle m(n)\rangle$, as a function of $n$, will initially grow as $\sim \sqrt{\frac{2 n}{\pi}}$ (the critical behaviour at $r=0$ ), before crossing over at a characteristic time $n^{*}(r) \sim 1 / r$ to the off-critical linear growth, $\langle m(n)\rangle \sim \sqrt{r / 2} n$. In Figure 4 , we compare the numerical simulation results for small values of $r$ and show how they approach the analytical scaling function $f_{m}(y)$ as $r \rightarrow 0$.

Next we consider the variance of the maximum, $\sigma_{m}^{2}=$ $\left\langle m^{2}(n)\right\rangle-\langle m(n)\rangle^{2}$ in the scaling limit $r \rightarrow 0, n \rightarrow \infty$ but keeping the product $r n$ fixed. For this, we now need to analyze the second moment in Eq. (76) in the scaling limit. The analysis proceeds more or less as in the case of the mean. We do not repeat this computation here and just mention the final result. In the scaling limit $r \rightarrow 0, n \rightarrow \infty$ with the product $r n$ fixed, we find that the variance behaves as

$$
\sigma_{m}^{2} \approx n F_{m}(r n),
$$

with the scaling function $F_{m}(y)$ given by

$$
F_{m}(y)=1+\frac{y}{2}-f_{m}^{2}(y),
$$

where $f_{m}(y)$ is given in Eq. (124). The scaling function has the following asymptotic behaviors

$$
F_{m}(y) \rightarrow \begin{cases}1-\frac{2}{\pi} & \text { as } y \rightarrow 0, \\ \frac{1}{2} & \text { as } y \rightarrow \infty .\end{cases}
$$

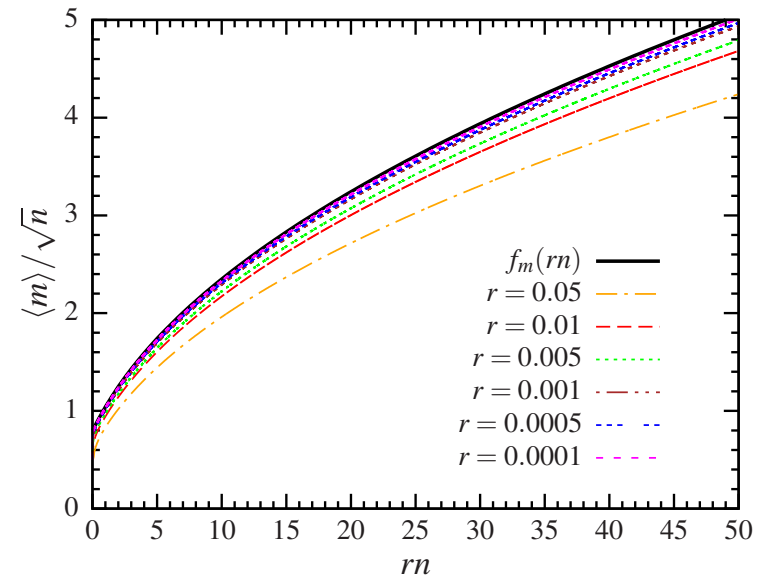

FIG. 4. (Color online) $\frac{\langle m(n)\rangle}{\sqrt{n}}$ plotted vs. $y=r n$ for different small values of $r$. As $r \rightarrow 0$, the curves approach the analytical scaling function $f_{m}(y)$ in Eq. 124 plotted as a solid line.

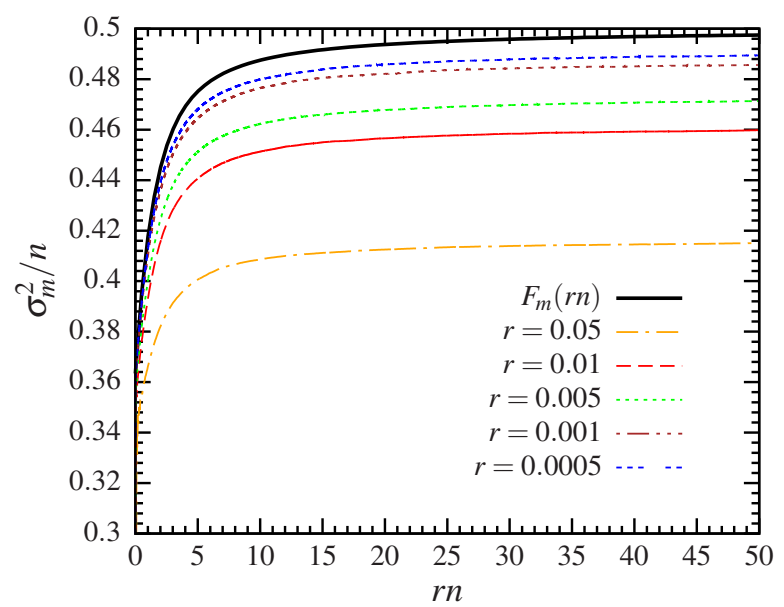

FIG. 5. (Color online) $\sigma_{m}^{2} / n$ plotted vs. $y=r n$ for different small values of $r$. As $r \rightarrow 0$, the curves approach the analytical scaling function $F_{m}(y)$ in Eq. 127) plotted as a solid line.

Hence, for $r=0$, using $F_{m}(0)=1-\frac{2}{\pi}$ in Eq. 126 gives the asymptotic behavior of the variance, $\sigma_{m}^{2} \simeq\left(1-\frac{2}{\pi}\right) n$, i.e., the result for normal diffusion without resetting. In contrast, for $r>0$, as $n \rightarrow \infty$, the scaling argument $y \rightarrow \infty$. Hence, using $F_{m}(y) \rightarrow 1 / 2$ as $y \rightarrow \infty$ in Eq. 126 gives, $\sigma_{m}^{2} \simeq n / 2$. This agrees perfectly with the finite $r$ result, $\sigma_{m}^{2} \simeq D(r) n$ with $D(r)$ given in Eq. 10 , since $D(r \rightarrow 0)=1 / 2$. The exact scaling function $F_{m}(y)$ thus interpolates smoothly between these two limits. In Figure 5, we compare the numerical simulation results with the analytical scaling function $F_{m}(y)$ in Eq. (127). 


\section{B. Scaling functions associated with the maximum $x(n)$}

We now turn to the scaling behavior of the mean and the variance of the position $x(n)$. We start with the mean whose exact generating function is given in Eq. (89). To analyze the scaling limit $r \rightarrow 0, n \rightarrow \infty$ while keeping the product $y=r n$ fixed, we follow the same procedure as in the case of the maximum. Setting $\lambda=1-p$ with $p \rightarrow 0$, and using Eq. (119), we find that Eq. (89) reduces, in the scaling limit, to the following integral

$$
\int_{0}^{\infty}\langle x(n)\rangle e^{-p n} d n \approx \frac{r}{p^{2} \sqrt{2(r+p)}} .
$$

It then indicates the following scaling behavior for the mean position

$$
\langle x(n)\rangle \approx \sqrt{n} f_{x}(r n),
$$

where $f_{x}(y)$, using Eq. [129,, satisfies

$$
\int_{0}^{\infty} \sqrt{y} f_{x}(y) e^{-s y} d y=\frac{1}{s^{2} \sqrt{2(1+s)}}
$$

One can again easily invert the Laplace transform in Eq. (131) to get

$$
f_{x}(y)=\frac{1}{\sqrt{2 y}}\left[\left(y-\frac{1}{2}\right) \operatorname{erf}(\sqrt{y})+\sqrt{\frac{y}{\pi}} e^{-y}\right] .
$$

It has the asymptotics

$$
f_{x}(y) \sim \begin{cases}\frac{2}{3} \sqrt{\frac{2}{\pi}} y+O\left(y^{2}\right) & \text { as } y \rightarrow 0 \\ \sqrt{\frac{y}{2}}+O\left(\frac{1}{\sqrt{y}}\right) & \text { as } y \rightarrow \infty .\end{cases}
$$

When $r=0$, using $f_{x}(0)=0$, one recovers the standard random walk (without resetting) result, $\langle x(n)\rangle=0$. In contrast, for $r>0$, when $n \rightarrow \infty$, i.e., the product $y=r n \rightarrow \infty$, using the large $y$ asymptotic behavior in Eq. (133), one gets, $\langle x(n)\rangle \simeq \sqrt{r / 2} n$, compatible with the linear growth with speed $v(r)$ in Eq. (16) upon noting that $v(r \rightarrow 0)=\sqrt{r / 2}$. The scaling function $f_{x}(y)$ interpolates between these two limits. Figure 6 demonstrates how simulation results converge to the analytical scaling function $f_{x}(y)$ in Eq. 132 as $r \rightarrow 0$.

We next consider the scaling behavior of the variance of the position, $\sigma_{x}^{2}=\left\langle x^{2}(n)\right\rangle-\langle x(n)\rangle^{2}$, in the scaling limit $r \rightarrow 0$, $n \rightarrow \infty$ with the product $y=r n$ fixed. Here we analyze the generating function for the second moment in Eq. (90) in the scaling limit. Since the procedure is identical as in the case of the maximum, we skip the details and present only the result. We find that in the scaling limit, the variance of the position behaves as

$$
\sigma_{x}^{2} \approx n F_{x}(r n)
$$

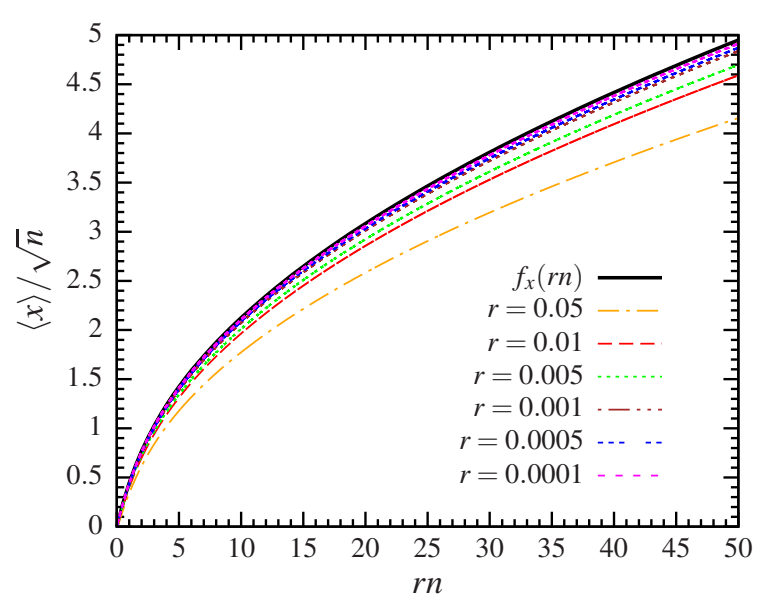

FIG. 6. (Color online) $\frac{\langle x(n)\rangle}{\sqrt{n}}$ plotted vs. $y=r n$ for different small values of $r$. As $r \rightarrow 0$, the curves approach the analytical scaling function $f_{x}(y)$ in Eq. 132 plotted as a solid line.

with the scaling function $F_{x}(y)$ given by

$$
F_{x}(y)=\frac{y}{2}+\frac{1-e^{-y}}{y}-f_{x}^{2}(y),
$$

where $f_{x}(y)$ is given in Eq. 132 . The scaling function $F_{x}(y)$ has the following asymptotic behaviors

$$
F_{x}(y) \rightarrow \begin{cases}1 & \text { as } y \rightarrow 0 \\ \frac{1}{2} & \text { as } y \rightarrow \infty\end{cases}
$$

Using these asymptotic behaviors, it is again easy to check that $F_{x}(y)$ smoothly interpolates between the critical $(r=0)$ and the off-critical $(r>0)$ behavior of the variance of the position, for finite but large $n$. In Figure 7, we compare the numerical simulation results with the analytical scaling function $F_{x}(y)$ in Eq. 135.

\section{CONCLUSION}

In conclusion, we have considered a model of random walk in one dimension where the walker, at each time step, resets to the maximum of the already visited positions with a certain probability $r$. For $r=0$, it reduces to the standard random walk in one dimension. The presence of a nonzero resetting probability $r$ changes drastically the asymptotic behavior of the walker. We find that on average, both the position and the maximum move with the same speed, and we have obtained an exact expression for this speed $v(r)$. The fluctuations about the mean is again described by the same diffusion coefficient $D(r)$ for both. We also obtain the large deviation form of the probabilities of finding the walker at a distance $y$ away from the maximum. The associated large deviation function shows a second order phase transition.

An interesting extension would be to study the walk which resets either to the maximum or the minimum with an equal 


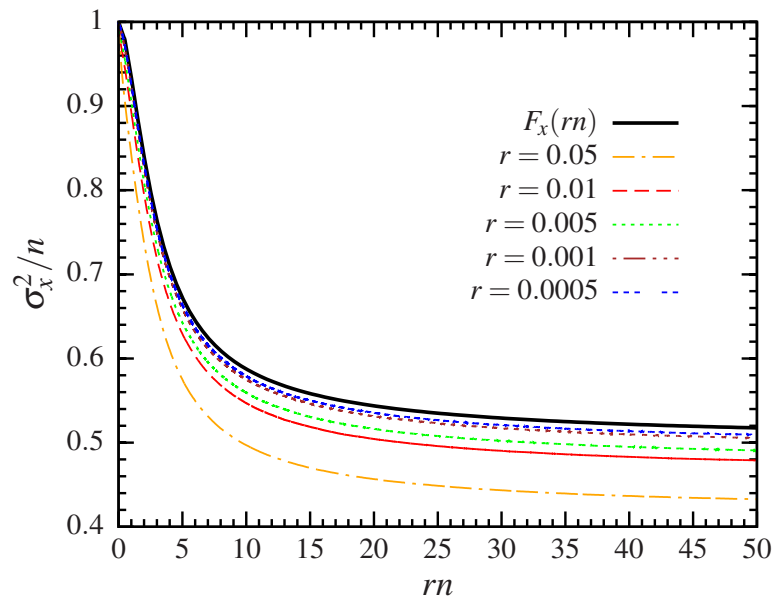

FIG. 7. (Color online) $\sigma_{x}^{2} / n$ plotted vs. $y=r n$ for different small values of $r$. As $r \rightarrow 0$, the curves approach the analytical scaling function $F_{x}(y)$ in Eq. 135 plotted as a solid line. probability $r / 2$. In general, one could ask the question in higher dimension, where the walker resets to one of the boundary sites of the already visited sites. Yet another extension, beyond the study of the fluctuations of the position and the maximum studied here, concerns the study of the search process with resetting to the maximum. For instance, it would be interesting to compute the mean first-passage time to an immobile or a moving target for a such a random walker submitted to random resetting to the maximum.

\section{ACKNOWLEDGMENTS}

The authors acknowledge the support of the Indo-French Centre for the Promotion of Advanced Research (IFCPAR/CEFIPRA) under Project 4604-3. S. N. M acknowledges useful discussions with P. Sen and her hospitality at the physics department of Calcutta University, where part of this work was done during a visit in July, 2015.
[1] G. M. Viswanathan, V. Afanasyev, S. V. Buldyrev, E. J. Murphy, P. A. Prince, and H. E. Stanley, Nature 381, 413 (1996).

[2] G. M. Viswanathan, S. V. Buldyrev, S. Havlin, M. G. E. da Luz, E. P. Raposo, and H. E. Stanley, Nature 401, 911 (1999).

[3] A. M. Edwards, R. A. Phillips, N. W. Watkins, M. P. Freeman, E. J. Murphy, V. Afanasyev, S. V. Buldyrev, M. G. E. da Luz, E. P. Raposo, H. E. Stanley, and G. M. Viswanathan, Nature 449, 1044 (2007).

[4] O. Bénichou, C. Loverdo, M. Moreau, and R. Voituriez, Rev. Mod. Phys. 83, 81 (2011).

[5] M. G. E. D. Luz, A. Grosberg, E. P. Raposo, and G. M. Viswanathan (editors), special issue: The Random Search Problem: Trends And Perspectives, J. Phys. A: Math. and Theor. 42, 430301-434017 (2009).

[6] S. C. Manrubia and D.H. Zanette, Phys. Rev. E 59, 4945 (1999).

[7] E. Gelenbe, Phys. Rev. E 82, 061112 (2010).

[8] M. Montanari and R. Zecchina, Phys. Rev. Lett. 88, 178701 (2002).

[9] S. Janson and Y. Peres, SIAM J. Discrete Math. 26, 537 (2012).

[10] M. R. Evans and S. N. Majumdar, Phys. Rev. Lett. 106, 160601 (2011).

[11] S. N. Majumdar, S. Sabhapandit, and G. Schehr, Phys. Rev. E 91, 052131 (2015).

[12] M. R. Evans and S. N. Majumdar, J. Phys. A: Math. Theor. 47, 285001 (2014).

[13] M. R. Evans, S. N. Majumdar, and K. Mallick, J. Phys. A: Math. Theor. 46, 185001 (2013).
[14] M. R. Evans and S. N. Majumdar, J. Phys. A: Math. Theor. 44, 435001 (2011).

[15] J. Whitehouse, M. R. Evans, and S. N. Majumdar, Phys. Rev. E 87, 022118 (2013).

[16] M. Montero and J. Villarroel, Phys. Rev. E 87, 012116 (2013).

[17] L. Kusmierz, S. N. Majumdar, S. Sabhapandit, and G. Schehr, Phys. Rev. Lett. 113, 220602 (2014).

[18] L. Kusmierz, and E. Gudowska-Nowak, preprint arXiv:1508.03184.

[19] C. Christou and A. Schadschneider, J. Phys. A: Math. Theor. 48, 285003 (2015).

[20] A. Pal, Phys. Rev. E 91, 012113 (2015).

[21] D. Boyer and C. Solis-Salas, Phys. Rev. Lett. 112, 240601 (2014).

[22] S. Reuveni, M. Urbach, and J. Klafter, Proc. Natl. Acad. Sci. USA 111, 4391 (2014).

[23] S. Gupta, S. N. Majumdar, and G. Schehr, Phys. Rev. Lett. 112, 220601 (2014).

[24] X. Durang, M. Henkel, and H. Park, J. Phys. A: Math. Theor. 47045002 (2014).

[25] H. C. Berg, Random Walks in Biology (Princeton University Press, New York, 1983); L. Edelstein-Keshet, Math- ematical Models in biology (McGraw Hill, Boston, 1988).

[26] F. Bartumeus et. al., Ecology, 86, 3078 (2005).

[27] R. Rajesh and S. N. Majumdar, J. Stat. Phys. 99, 943 (2000).

[28] R. Rajesh and S. N. Majumdar, Phys. Rev. E 62, 3186 (2000).

[29] R. Rajesh and S. N. Majumdar, Phys. Rev. E 64, 036103 (2001).

[30] S. Sabhapandit, Phys. Rev. E 85, 021108 (2012). 